\title{
Preclinical and Clinical Studies Demonstrate That the Proprietary Herbal Extract DA-5512 Effectively Stimulates Hair Growth and Promotes Hair Health
}

\author{
Jae Young Yu, ${ }^{1}$ Biki Gupta, ${ }^{2}$ Hyoung Geun Park, ${ }^{1}$ Miwon Son, ${ }^{1}$ Joon-Ho Jun, ${ }^{1}$ \\ Chul Soon Yong, ${ }^{2}$ Jeong Ah Kim, ${ }^{3}$ and Jong Oh Kim ${ }^{2}$ \\ ${ }^{1}$ Dong-A Pharm Research Institute, Pharmaceutical Product Research Laboratories, Yongin 449-905, Republic of Korea \\ ${ }^{2}$ College of Pharmacy, Yeungnam University, 214-1 Daedong, Gyeongsan 712-749, Republic of Korea \\ ${ }^{3}$ College of Pharmacy, Research Institute of Pharmaceutical Sciences, Kyungpook National University, Daegu 41566, Republic of Korea
}

Correspondence should be addressed to Jeong Ah Kim; jkim6923@knu.ac.kr and Jong Oh Kim; jongohkim@yu.ac.kr

Received 25 January 2017; Accepted 3 April 2017; Published 30 April 2017

Academic Editor: Darren R. Williams

Copyright @ 2017 Jae Young Yu et al. This is an open access article distributed under the Creative Commons Attribution License, which permits unrestricted use, distribution, and reproduction in any medium, provided the original work is properly cited.

\begin{abstract}
The proprietary DA-5512 formulation comprises six herbal extracts from traditional oriental plants historically associated with therapeutic and other applications related to hair. Here, we investigated the effects of DA-5512 on the proliferation of human dermal papilla cells (hDPCs) in vitro and on hair growth in C57BL/6 mice and conducted a clinical study to evaluate the efficacy and safety of DA-5512. DA-5512 significantly enhanced the viability of hDPCs in a dose-dependent manner $(p<0.05)$, and 100 ppm of DA-5512 and $1 \mu \mathrm{M}$ minoxidil (MXD) significantly increased the number of Ki-67-positive cells, compared with the control group $(p<0.05)$. MXD (3\%) and DA-5512 (1\%, 5\%) significantly stimulated hair growth and increased the number and length of hair follicles (HFs) versus the controls (each $p<0.05$ ). The groups treated with DA-5512 exhibited hair growth comparable to that induced by MXD. In clinical study, we detected a statistically significant increase in the efficacy of DA-5512 after 16 weeks compared with the groups treated with placebo or $3 \% \mathrm{MXD}(p<0.05)$. In conclusion, DA-5512 might promote hair growth and enhance hair health and can therefore be considered an effective option for treating hair loss.
\end{abstract}

\section{Introduction}

Alopecia or hair loss is a dermatologic disease, which affects men and women, and often affects self-esteem and personal attractiveness, potentially leading to depression and other negative effects [1]. Alopecia is caused by nutritional, autoimmune, or environmental factors [2-4]. A striking observation of people with alopecia is that, despite their poor quality of life, most patients do not pursue therapy, mainly because of the poor efficacy of available treatment approaches, the associated adverse effects, tolerance to treatment, and lack of proper information.

Minoxidil (MXD) is a potassium channel opener and vasodilator that is widely recommended to treat androgenetic alopecia, which increases the duration of "anagen" and enlarges miniaturized and suboptimal follicles. However, the mechanism of action of MXD, which stimulates hair growth, is not well understood [5-8]. Despite the perceived advantages of MXD for treating alopecia, it causes numerous dermatologic adverse effects such as scalp irritation, dryness, sealing, itching, erythema, and contact dermatitis [9-11].

Finasteride is a type- 2 inhibitor of 3 -oxo- $5 \beta$-steroid 4 dehydrogenase ( $5 \alpha$-reductase), which was studied as a treatment for male or female pattern baldness. Although there is limited evidence of the efficacy of finasteride, it may be considered a treatment option for patients who fail to respond to topical MXD [12]. Finasteride is well tolerated; and $2.5 \mathrm{mg}$ daily seems to achieve better results compared with $1.0 \mathrm{mg}$ daily. The adverse effects of finasteride include decreased libido and erectile dysfunction in men, and finasteride is contraindicated for treating pregnant women because of its teratogenicity [13]. Despite the efficacy of these drugs under certain circumstances, their long-term effects and side effects 
TABLE 1: Plants used to prepare herbal extracts of the proprietary formulation DA-5512.

\begin{tabular}{lccc}
\hline Subject & Custom & Identifier & Local area \\
\hline Thea sinensis L. & Soa Dawon herb & Myung-hae Choi & Bosung Jeonnam \\
Tribulus terrestris & Hong-il dang & CK Trading Company & North India Punjab \\
Pinus densiflora & The one herb & Hong-geun Kim & Chuncheon, Gangwon-do \\
Emblica officinalis & Duc-su mu yuck & Bayir Chemical Company & Bangalore \\
Pueraria thunbergiana & The one herb & Hansin Logis Company & Guangxi Zhuangzu \\
Zingiber officinale & The one herb & Chunha Company & Shanding \\
\hline
\end{tabular}

remain problematic. Therefore, researchers have intensified their interest in evaluating alternative remedies such as herbal medicines and products. Several herbal formulations are commercially available to treat alopecia, such as hair tonics, hair-growth stimulants, hair conditioners, hair-cleansing agents, and antidandruff agents [14].

The proprietary DA-5512 formulation includes herbal extracts from Thea sinensis L., Emblica officinalis, Pinus densiflora, Pueraria thunbergiana, Tribulus terrestris, and Zingiber officinale. All sources are traditional oriental plants that have long been used for the therapy of ailments related to hair. Thea sinensis L. (green tea) has potential beneficial effects because of the anticancer and antioxidant properties of its component epigallocatechin-3-gallate (EGCG), a major constituent of polyphenols [15]. Moreover, EGCG promotes hair growth by stimulating proliferation and inhibiting apoptosis of hair dermal papilla cells [16].

Emblica officinalis (Indian gooseberry or Amla), which is an important herbal component of Thai traditional recipes, is believed to slow aging. Moreover, Amla acts as a diuretic, laxative, liver tonic, refrigerant, stomachic, restorative, alterative, antipyretic, anti-inflammatory, and hair tonic as well as an antiulcer, antidyspeptic, and digestive agent [17]. A fixed oil is obtained from these gooseberries, which is used to strengthen and promote hair growth. The dried fruit, which improves hair hygiene, has long been utilized as an important ingredient of shampoo and hair oil [18]. Thus, the Indian gooseberry is used as a hair tonic in traditional recipes for enriching hair growth and pigmentation.

Phyllanthus emblica L. is a potent inhibitor of $5 \alpha$-reductase, which promotes the growth of the hair of C57BL/ 6 mice [19]. The pine needle (Pinus densiflora Sieb \& Zucc.) is commonly used as a herbal medicine in East Asian countries. Pine needle tea is high in vitamin A, beta-carotene, and vitamin $C$, which prevent oxidative stress in the skin and help to keep skin looking young by eliminating free radicals. Vitamins A and C help prevent hair loss and dandruff as well [20]. Pueraria thunbergiana (kudzu) is a climbing plant of the Leguminosae family, which is native to China, Japan, and Korea.

The roots and flowers of $P$. thunbergiana are used in traditional medicine because of their medicinal properties $[21,22]$, and a study of a murine model of hair loss demonstrates their antiandrogenic activity and proliferative effects on hair growth [19]. Similarly, the rhizome of the widely used member of the ginger family Zingiber officinale has been used for thousands of years to enhance the flavor and aroma of food. Further, Z. officinale rhizomes are a source of antimicrobials as well as nonsteroidal anti-inflammatory drugs [23]. Ginger is a powerful antifungal agent that is used in shampoo to treat dandruff [24]. The antihypertensive effects of fruits of $T$. terrestris are similar to those of MXD, and its beneficial effects are attributed in part to its ability to release nitric oxide from the endothelium and nitrergic nerve endings [25]; however, there are no reports of its effects on human hair growth. The present study was undertaken to investigate the pharmacological effects of DA-5512 on the proliferation of human dermal papilla cells (hDPCs) in vitro and its effects on C57BL/6 mice and to evaluate its efficacy and safety when administered to human subjects.

\section{Materials and Methods}

2.1. Materials. The plants listed in Table 1 , which were purchased locally or from farms located in India and China, were ultrasonically extracted in $100 \%$ ethanol at room temperature for 3 days. The extracts were concentrated using a rotary vacuum evaporator (Eyela N-N, Tokyo, Japan), and the mixed concentrates were collected and sterilized for $10 \mathrm{~min}$. The sterilized concentrates were filtered twice and then analyzed using high-performance liquid chromatography (HPLC) to determine their phytochemical contents as described in the next section.

2.2. Phytochemical Analysis. The HPLC apparatus (Infinity 1290 System; Agilent, Mississauga, Ontario, Canada) was equipped with an Eclipse Plus $\mathrm{C}_{18}$ column $(150 \mathrm{~mm} \times 2.1 \mathrm{~mm}$ id, $1.8 \mu \mathrm{m}$; Agilent, Mississauga, Ontario, Canada). The column was eluted with a gradient formed by water and formic acid (0.1\%) (solvent A) and acetonitrile (solvent B). The initial mobile phase contained $98 \%$ solvent $\mathrm{A}$ and $2 \%$ solvent B and was delivered for $10.0 \mathrm{~min}$, and then solvent B was increased to $50 \%$ and then to $90 \%$ after $30.0 \mathrm{~min}$ and $40.0 \mathrm{~min}$, respectively, and was then equilibrated for $10 \mathrm{~min}$ using the initial conditions. The flow rate was $0.35 \mathrm{~mL} / \mathrm{min}$, the column temperature was $40^{\circ} \mathrm{C}$, the detection wavelength was $254 \mathrm{~nm}$, and the injection volume was $1 \mu \mathrm{L}$.

\subsection{Effect of DA-5512 on the Proliferation of hDPCs}

2.3.1. Isolation and Culture of hDPCs. Human occipital scalp skin specimens from hair transplantation surgery were obtained after receiving the patients' informed consent. The Institutional Ethics Committee of Yonsei University, 
Wonju College of Medicine, Wonju, Korea, approved this study, which was conducted according to guidelines of the Declaration of Helsinki and Tokyo for humans. Samples were dissected into single HFs, and human dermal papillae were obtained from individually isolated HFs as previously reported [26, 27]. Isolated hDPCs were transferred to a plastic dish and cultured in Dulbecco's modified Eagle's medium (DMEM; Gibco BRL, Gaithersburg, MD, USA) supplemented with penicillin $(100 \mathrm{IU} / \mathrm{mL})$, streptomycin $(100 \mu \mathrm{g} / \mathrm{mL}$ ), and $10 \%$ fetal bovine serum (FBS) (HyClone, Logan, UT, USA) in a humidified incubator set to $37^{\circ} \mathrm{C}$ that contained an atmosphere of $5 \% \mathrm{CO}_{2}$. Second and third passage hDPCs were used.

2.3.2. Viability Assay. Cell viabilities were determined using 3-[4,5-dimethylthiazol-2-yl]-2,5 diphenyl tetrazolium bromide (MTT) [28]. Briefly, $1 \times 10^{4}$ cells in $100 \mu \mathrm{L}$ of growth medium were seeded into each well of a 96-well plate, allowed to adhere for $24 \mathrm{~h}$, and then treated with serial doses of DA-5512 (0-10,000 ppm) or $1 \mu \mathrm{M}$ MXD (positive control) (Sigma-Aldrich, St. Louis, MO, USA) for 24 and $48 \mathrm{~h}$, respectively [29]. After treatment, the medium in each well was removed and replaced with phosphate-buffered saline (PBS) containing $5 \mathrm{mg} / \mathrm{mL}$ MTT, and the plate was then incubated at $37^{\circ} \mathrm{C}$ for $4 \mathrm{~h}$. The remaining supernatant was then removed, and $100 \mu \mathrm{L}$ of DMSO was added to each well and mixed thoroughly to dissolve the crystallized formazan. To ensure that all formazan crystals were completely dissolved after $10 \mathrm{~min}$ incubation, the optical density at $540 \mathrm{~nm}$ was determined using an ELISA reader. Cell viability $(\%)=($ mean test absorbance) $/($ mean control absorbance $) \times 100$.

2.3.3. Proliferation Assay. Ki-67 expression was determined as a marker of cell proliferation. For this purpose, hDPCs were incubated on an $18 \mathrm{~mm}$ coverslip for $24 \mathrm{~h}$ in the presence of $100 \mathrm{ppm}$ DA-5512 or $1 \mu \mathrm{M}$ MXD. The cells were then washed twice with PBS, fixed in $4 \%$ paraformaldehyde in PBS for $15 \mathrm{~min}$ at $37^{\circ} \mathrm{C}$, permeabilized with $0.25 \%$ triton X-100 in PBS for $5 \mathrm{~min}$, and blocked with 1\% BSA in PBS for $30 \mathrm{~min}$. The cells were incubated with an anti-Ki-67 antibody (1:100 dilution) (Abcam, Cambridge, MA, USA) overnight at $4^{\circ} \mathrm{C}$, followed by incubation with Alexa Fluor 594-conjugated goat anti-rabbit IgG (1:200 dilution) for $1 \mathrm{~h}$ at room temperature. The hDPCs were then washed and counterstained with $1 \mu \mathrm{g} / \mathrm{mL}$ DAPI for $5 \mathrm{~min}$ and mounted on a glass slide. Images of fluorescence were acquired using a confocal laser scanning microscope (TCS SPE; Leica Microsystems GmbH, Wetzlar, Germany).

\subsection{Analysis of the Effect of DA-5512 on the Growth of Hair in C57BL/6 Mice}

2.4.1. Animals and Treatments. Seven-week-old male C57BL/6 mice were purchased from Daehan Biolink, Inc. (Eumseong, Korea). The animals were housed under conventional conditions that included a standard diet, ad libitum water, and a $12 \mathrm{~h}$ light cycle. Animals were first acclimatized for 1 week, and 20 mice were randomly separated into four groups of five mice each. Group 1, which served as the negative control (NC), was topically given $30 \%$ ethanol, and Group 2, which served as the positive control (PC), received 3\% topical MXD (Minoxil ${ }^{\circledR}$, Hyundai Pharm., Korea) hair tonic. Groups 3 and 4 received $1 \%$ and 5\% topical DA-5512 hair tonic, respectively. The hair on the back of each mouse was shaved with an electric clipper, avoiding injury or stimulation of the skin, which was then followed by application of a depilatory cream to clean the remaining hair. The mice were then allowed to rest for $24 \mathrm{~h}$, and $0.2 \mathrm{~mL}$ of the specified substance was then topically applied to the shaven areas daily for 14 days. Hair regrowth was examined and digital images were acquired using a Nikon Cool Pix P100 (Tokyo, Japan) on days 1, 7, 10, and 14. Changes in the area of hair regrowth (\%) within the shaven area were evaluated using ZoomBrowser software (Canon, Japan). For determination of hair weight and length, 30 hairs were picked randomly from the shaven area of each mouse on day 14 , and the weights and lengths of the hairs were recorded.

2.4.2. Hematoxylin and Eosin (HßE) Staining. $H \& E$ staining was employed to determine the effects of DA- 5512 on HFs. The mice were sacrificed upon completion of the study. Their dorsal skin was removed, fixed in $10 \%$ formaldehyde solution, and embedded in paraffin. Skin sections $(2-3 \mu \mathrm{m})$ were stained with $\mathrm{H} \& \mathrm{E}$, and follicular lengths and numbers as well as follicular morphologies were observed using a light microscope (Olympus, Tokyo, Japan).

2.4.3. Statistical Analyses of the Mouse Data. Statistical analyses were performed using one-way ANOVA followed by Dunnett's test. The data are reported as the mean \pm SD.

\subsection{Treatment of Hair Loss and Promotion of Scalp Health in Patients with Alopecia}

2.5.1. Study Subjects. The subjects included adults aged 20 to 60 years who were diagnosed with mild alopecia. Men and women were categorized according to the NorwoodHamilton or Ludwig classifications, respectively. Participants showed no evidence of skin disorders, had no history of cardiovascular, renal, or hepatic disease, and did not admit to applying any topical treatment drug (steroids, cytotoxic agents, vasodilators, antihypertensives, anticonvulsants, $\beta$ blockers, and diuretics) or any of the following agents: spironolactone, cimetidine, diazoxide, cyclosporine, ketoconazole, or replacement hormones. Subjects were excluded if they underwent surgery for hair loss, such as a hair transplant. Pregnant and lactating women were excluded as well. The Institutional Review Board of Chung-Ang University Hospital approved this study (C2012223[918]), which was performed in the $\mathrm{P} \& \mathrm{~K}$ Hair Clinic Center.

2.5.2. Study Design. Changes in hair characteristics after 8 and 16 weeks of treatment were photographed and compared with baseline (Canon EOS 550D; Canon, Japan). An area of the scalp of each patient was defined before the first treatment and marked by a tattoo to locate the exact area after shaving. The investigator photographed the vertex and frontal area of the scalp. A randomized study design was utilized as follows: 


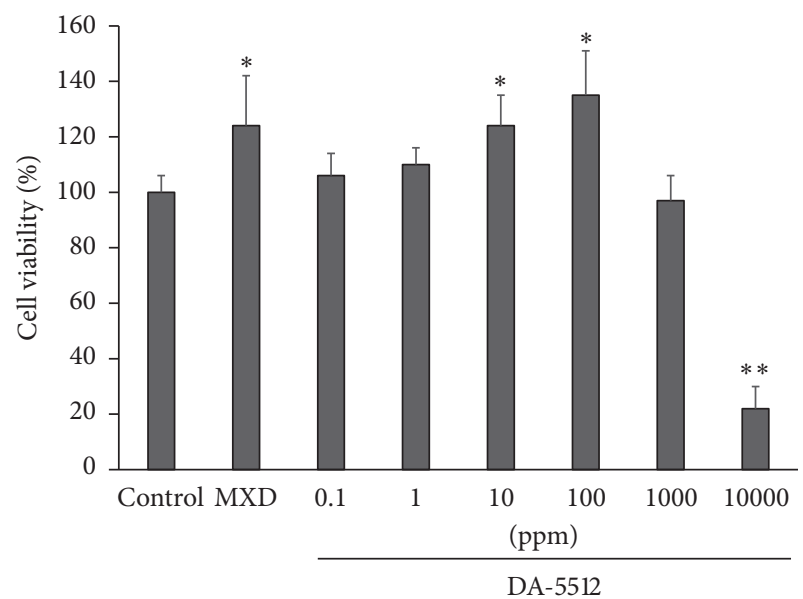

(a)

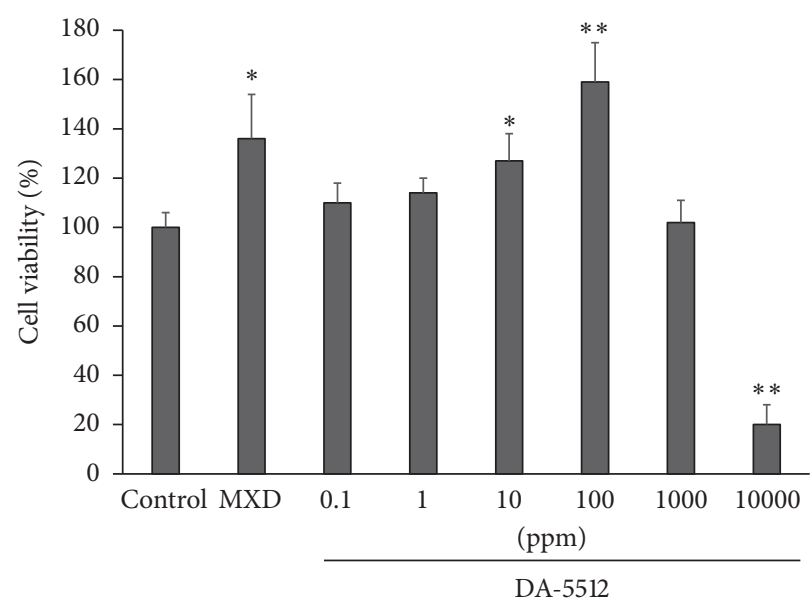

(b)

Figure 1: Viability of human dermal papilla cells after (a) $24 \mathrm{~h}$ and (b) $48 \mathrm{~h}$ treatment with various concentrations of DA-5512. Cell viability $(\%)=($ mean test absorbance $) /($ mean control absorbance $) \times 100$. Concentration of minoxidil $(\mathrm{MXD})$ was $1 \mu \mathrm{M}$. All values are expressed as the mean $\pm \operatorname{SD}(n=8) .{ }^{*} p<0.05$ and ${ }^{* *} p<0.01$ versus control, respectively.

5\% topical DA-5512 (Dong-A Pharmaceutical Co. Ltd., Korea) $(n=8) ; 3 \%$ topical MXD (positive control; capillus, DongA Pharmaceutical, Korea) $(n=7)$; or placebo (vehicle, $30 \%$ ethanol) $(n=8)$. Patients were given $1 \mathrm{~mL}$ of solution on the central scalp region twice daily at 12-h intervals for 16 weeks. The study comprised a baseline clinical visit (week 0 ) and two follow-up visits (weeks 8 and 16).

2.5.3. Study Endpoints. The primary efficacy endpoint included changes in hair density $\left(n / \mathrm{cm}^{2}\right)$, hair-shaft diameter $(\mathrm{mm})$, and hair-growth rate $(\mathrm{mm} /$ day) among the three groups. Hair density and hair-shaft diameter were assessed using phototrichogram software (Folliscope 4.0, Lead M, Seoul, Korea) at baseline and at 8 and 16 weeks of treatment. The investigator's assessment scores for hair density of each group were assigned, and an investigator uninformed of the nature of the study used a standardized 7-point rating scale for evaluating the changes in hair density acquired from photographs of the patients' scalps at these times. The scores $( \pm n)$ of the standardized 7-point rating scale were as follows: greatly decreased $(-3)$, moderately decreased $(-2)$, slightly decreased $(-1)$, unchanged $(0)$, slightly increased $(+1)$, moderately increased $(+2)$, and greatly increased $(+3)$. The secondary efficacy endpoint was the comparison of changes according to the investigator's assessment and the patients' self-assessments of the efficacy of the treatment according to hair growth, hair density, or satisfaction. For the latter, a 10-point scale was used as follows: 0 (lowest) to 10 (highest), and a patient's self-assessment of scalp condition used a standard 5-point rating scale as follows: 0 (greatly decreased) to 5 (greatly increased).

2.5.4. Safety. Safety monitoring was conducted through clinical evaluations and observations for adverse reactions experienced by any subjects.

2.5.5. Statistical Analysis for Clinical Study. Changes in parametric data from the study were analyzed using analysis of variance (ANOVA), and $p<0.05$ indicates statistical significance. The Wilcoxon signed rank test was used for nonparametric data obtained from patients' questionnaires after they used the products.

\section{Results}

3.1. Effects of DA-5512 on the Viability and Proliferation of hDPCs. DA-5512 and MXD $(1 \mu \mathrm{M})$ significantly enhanced the viability of hDPCs compared with the control group (each $p<0.05$ ) (Figure 1). Further, 10,000 ppm of DA-5512 was cytotoxic $(p<0.01)$. We found that $100 \mathrm{ppm}$ of DA-5512 and $1 \mu \mathrm{M}$ MXD significantly increased the number of Ki-67positive cells compared with the control group $(p<0.05)$ (Figure 2).

3.2. DA-5512 Promotes the Growth of the Hair of C57BL/6 Mice. Figure 3 demonstrates the hair-growth-promoting effects of DA-5512 on C57BL/ 6 mice after 14 days of treatment. NC-group mice exhibited only a faint appearance of hair after treatment for 14 days. The groups treated with PC (3\% MXD) and DA-5512 showed significant hair growth, which occupied the major portion of the back (Figure 3(a)). Digital image analysis of hair growth showed significantly higher values for the DA-5512-treated groups compared with that of the NC group and similar values compared with those of the PC group $(p<0.05)$ (Figure 3(b)). Notably, the 5\% DA-5512 dose group showed increased hair growth compared with that of the 1\% DA-5512 dose group. The mean hair lengths and weights on day 14 are shown in Figure 4. The DA-5512 and the 3\% MXD groups exhibited significant increases in mean hair length and weight on day $14(p<0.05)$. There were no significant differences in the measurements of hair weight and length between the 3\% MXD and 5\% DA-5512 groups.

To confirm the effect on the hair-growth cycle following the application of DA-5512, we analyzed the follicular morphology of the dorsal skin. Figure 5 demonstrates the 

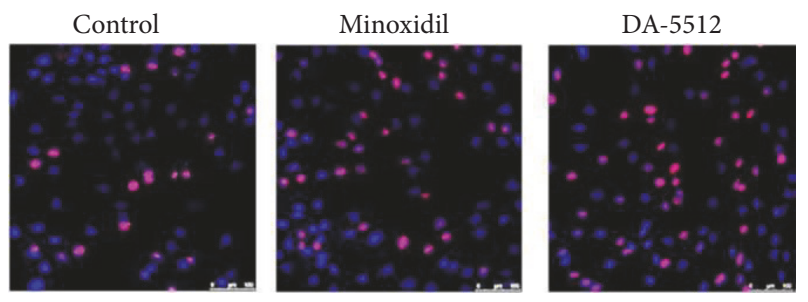

(a)

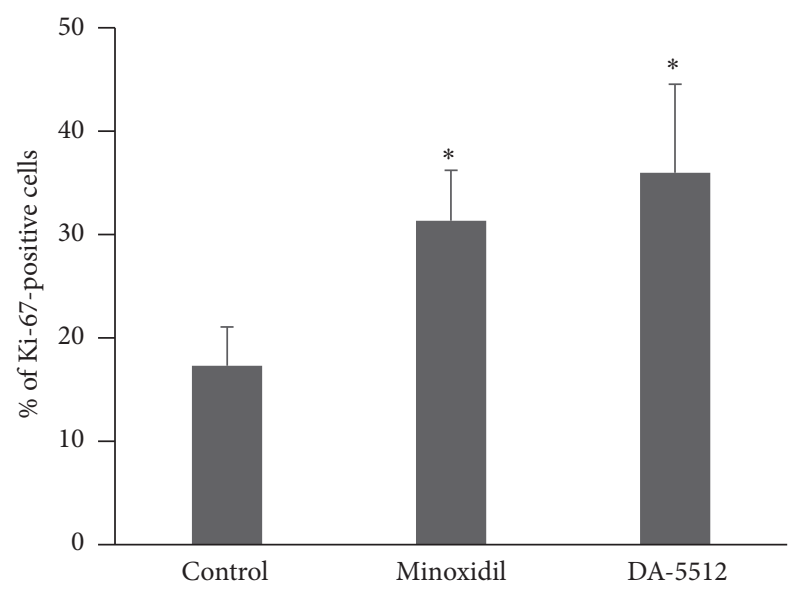

(b)

Figure 2: Effects of DA-5512 on Ki-67 levels in human dermal papilla cells. (a) The number of proliferating (Ki-67-positive, red) cells versus total hDPCs was determined. Nuclei were stained with DAPI (blue). Scale bar $=100 \mu \mathrm{m}$. (b) Ki-67-positive cells were counted in four nonoverlapping fields in each group and normalized to the total number of hDPCs (DAPI-positive). The concentrations of MXD and DA-5512 were $1 \mu \mathrm{M}$ and $100 \mathrm{ppm}$, respectively. All values are expressed as the mean $\pm \mathrm{SD} .{ }^{*} p<0.05$ versus control.

morphology, mean HF count, and mean HF length of each group. HF lengths and numbers of the 3\% MXD and DA-5512 groups differed significantly from those of the NC group $(p<$ 0.001 ) (Figure 5(b)). Mice given DA-5512 had significantly greater numbers of HFs compared with those of mice given the vehicle, but not compared with those of mice receiving $3 \%$ MXD.

\subsection{Stimulation of Hair Growth and Promotion of Hair Health by DA-5512 in Patients with Alopecia}

3.3.1. Age and Sex of the Study Subjects. Each of the three groups comprised at least 10 subjects. Two participants each from the DA-5512 and placebo groups and three participants from the 3\% MXD group resigned before completion of the study, and 23 participants completed the experiment. The percentages of men and women were $17.39 \%(4 / 23)$ and $82.61 \%(19 / 23)$, respectively. In the placebo and DA-5512 groups, the percentages of men and woman participants were $25 \%(2 / 8)$ and $75 \%(6 / 8)$, respectively. The percentages of men and women in the $3 \%$ MXD group were $0 \%(0 / 7)$ and $100 \%$ (7/7), respectively. There were no significant differences in the ages (years) of the three groups (DA-5512, $49.25 \pm 6.41 ; 3 \%$ MXD, $49.00 \pm 8.70$; placebo, $50.00 \pm 5.61)$.

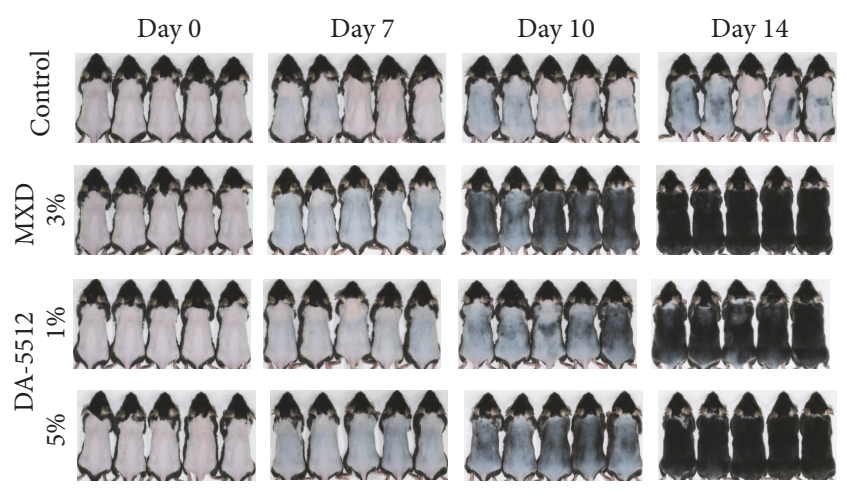

(a)

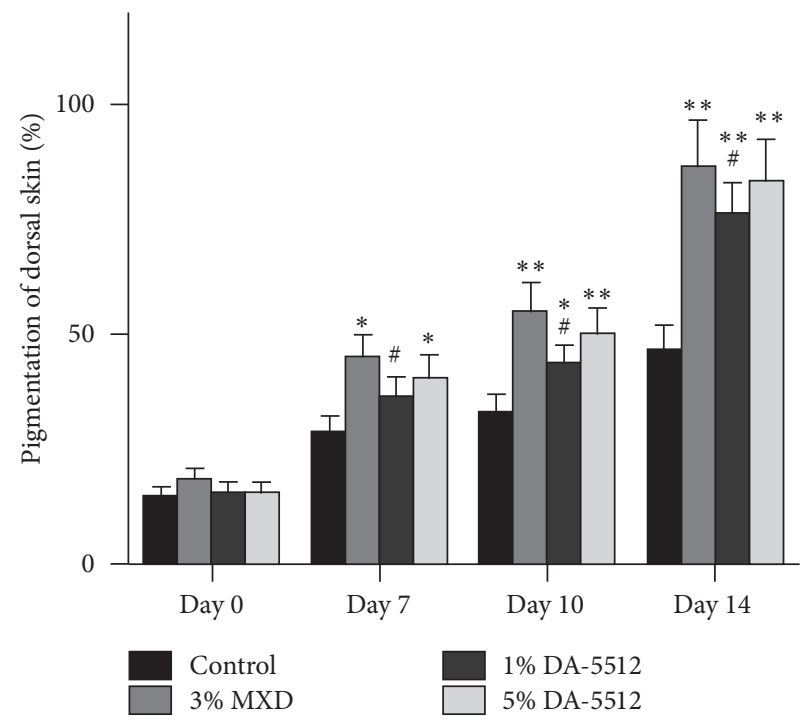

(b)

Figure 3: Hair-growth-promoting effects of DA-5512 on C57BL/6 mice. (a) Photographs of mice over 14 days of treatment with control (control, topical), 3\% topical MXD, and 1\% or 5\% topical DA-5512. (b) Quantified pigmentation of the dorsal skin (\%) in C57BL/6 mice over 14 days of treatment. ${ }^{*} p<0.05$ and $^{* *} p<0.001$ versus control, and ${ }^{\#} p<0.05$ versus $3 \%$ MXD.

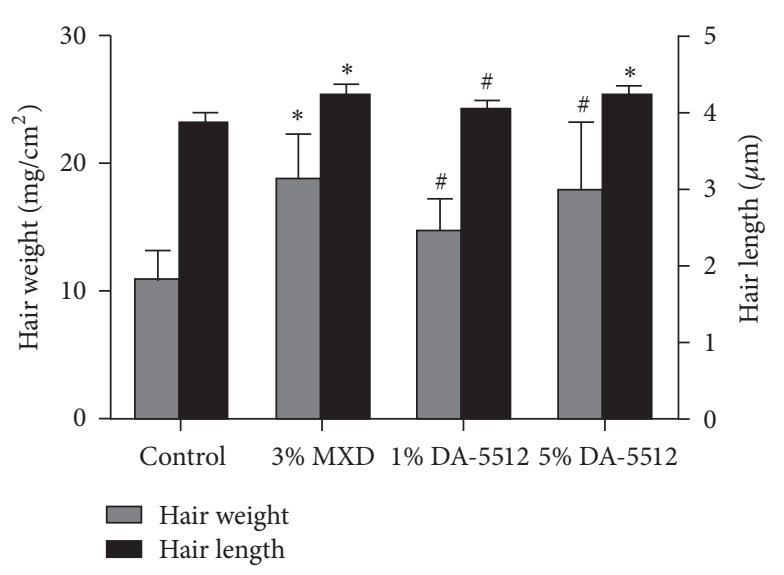

FIGURE 4: Hair length and weight of the dorsal skin of C57BL/6 mice after 14-day treatment. All values are expressed as the mean \pm SD. ${ }^{\#} p<0.05$ and ${ }^{*} p<0.01$ versus the control. 

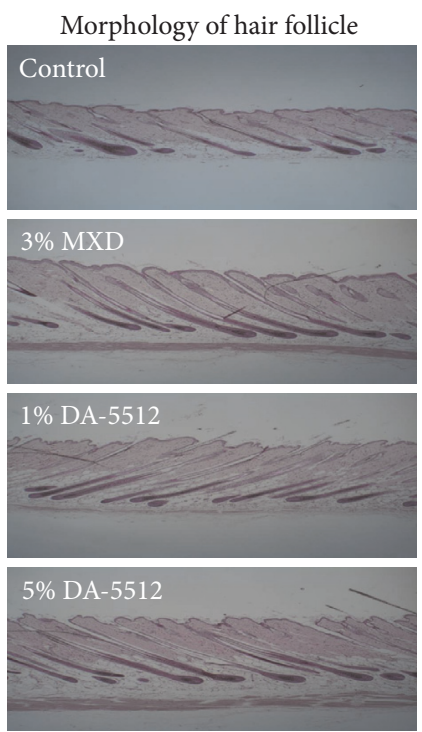

(a)

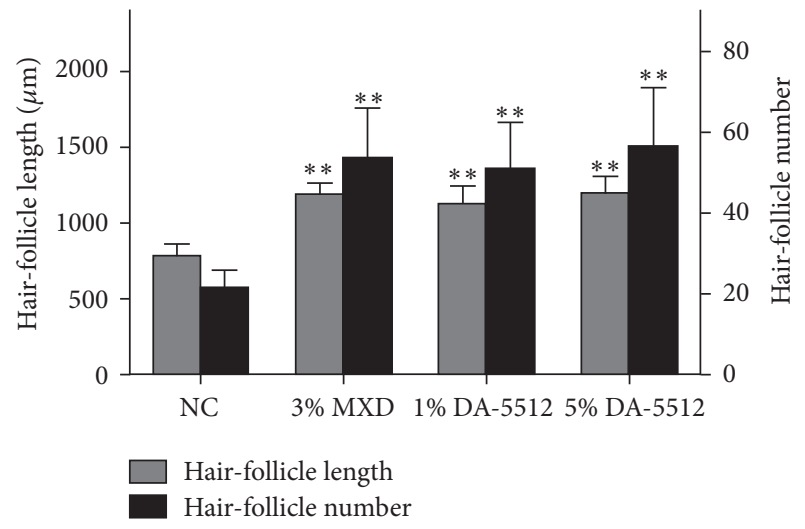

(b)

FIgURE 5: Histological analysis (H\&E staining) of the basal layer of the epidermis of C57BL/6 mice after 14 days of treatment. (a) Morphology and (b) length and number of hair follicles in the epidermis. Digital images were obtained using a light microscope (100× magnification). All values are expressed as the mean \pm SD. ** $p<0.001$ versus control.

3.3.2. Hair Density and Hair-Shaft Diameter. The changes in hair density and hair-shaft diameter determined using folliscope measurements over 16 weeks are shown in Figures 6 and 7 , respectively. The mean changes in hair density $\left(n / \mathrm{cm}^{2}\right)$ after 8 and 16 weeks of treatment with $3 \%$ MXD $(10.14 \pm 6.84$ and $14.29 \pm 5.74)$ and 5\% DA-5512 (7.75 \pm 7.66 and $6.62 \pm$ 4.89) were significantly higher $(p<0.01)$ compared with those treated with placebo $(-0.75 \pm 6.67$ and $-0.125 \pm 3.81)$. Similarly, the mean changes in hair-shaft diameter $(\mathrm{mm})$ after 8 and 16 weeks of treatment with $3 \% \operatorname{MXD}(0.007 \pm 0.006$ and $0.009 \pm 0.006$, resp. $)$ were significantly higher $(p<0.05)$ compared with those of the placebo group $(-0.002 \pm 0.009$ and $0.000 \pm 0.002)$. However, the mean changes in hairshaft diameter after 8 weeks of treatment with 5\% DA-5512 $(0.000 \pm 0.006)$ were not significant, while those after 16 weeks of treatment with $5 \%$ DA-5512 (0.006 \pm 0.003$)$ were

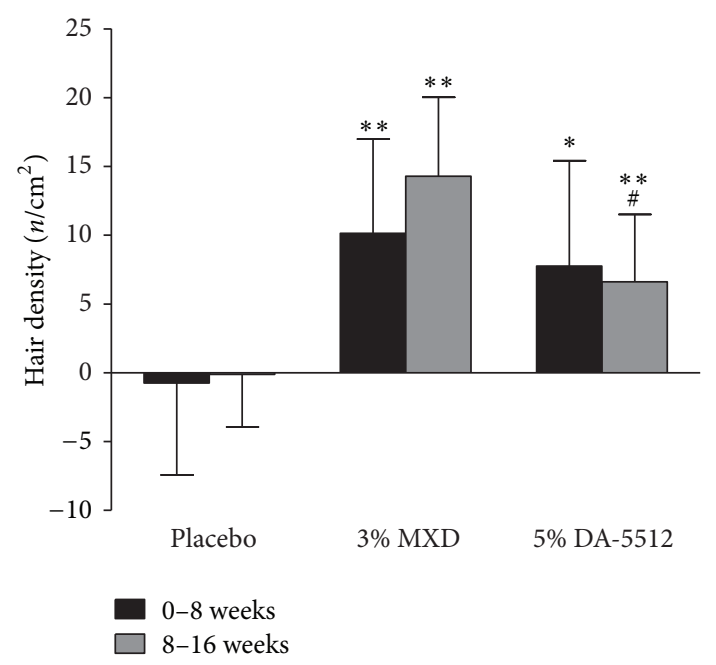

(a)

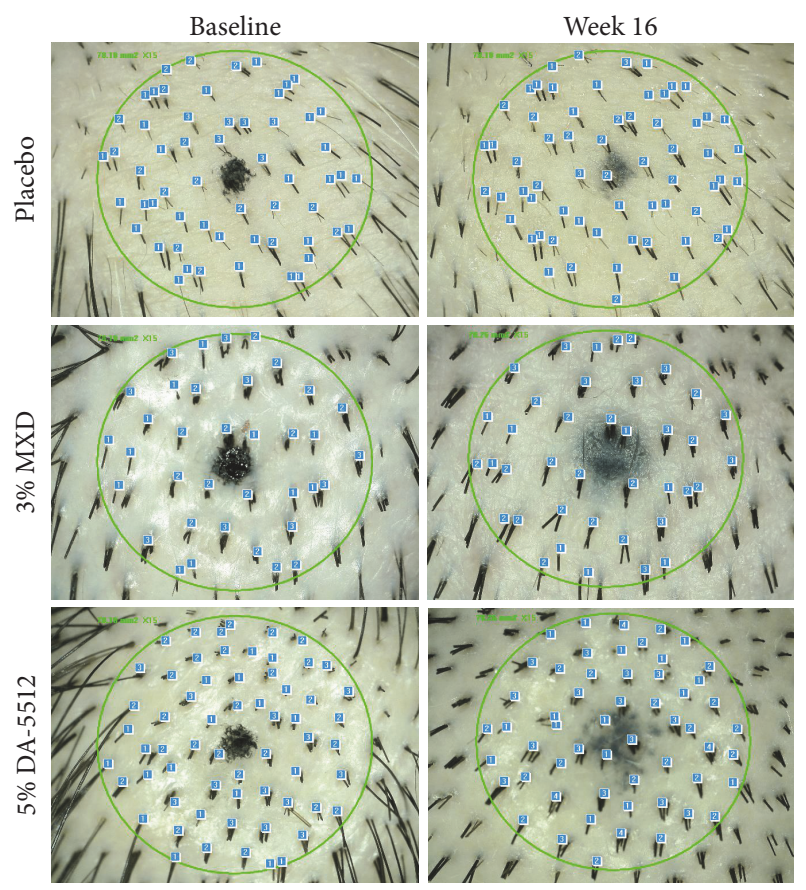

(b)

FIgURE 6: (a) Changes in hair density through days 0-8 and 8-16 days after treatment with placebo, 3\% MXD, or 5\% DA-5512. All values are expressed as the mean \pm SD. ${ }^{*} p<0.05$ and ${ }^{* *} p<0.01$ versus placebo, and ${ }^{\#} p<0.05$ versus $3 \%$ MXD. (b) Folliscopy of hair density at baseline and week 16 after treatment with placebo, $3 \%$ MXD, or 5\% DA-5512.

significantly higher $(p<0.05)$ compared with those of the placebo group. The mean changes in diameter following treatment with 3\% MXD were significantly higher compared with treatment with 5\% DA-5512 after 8 weeks $(p<0.05)$, although the difference was not statistically significant after 16 weeks.

3.3.3. Hair-Growth Rate. The mean hair-growth rates ( $\mathrm{mm} /$ day) of five strands of hair in the target area after 8 and 16 weeks of treatment with 3\% MXD and 5\% DA-5512 


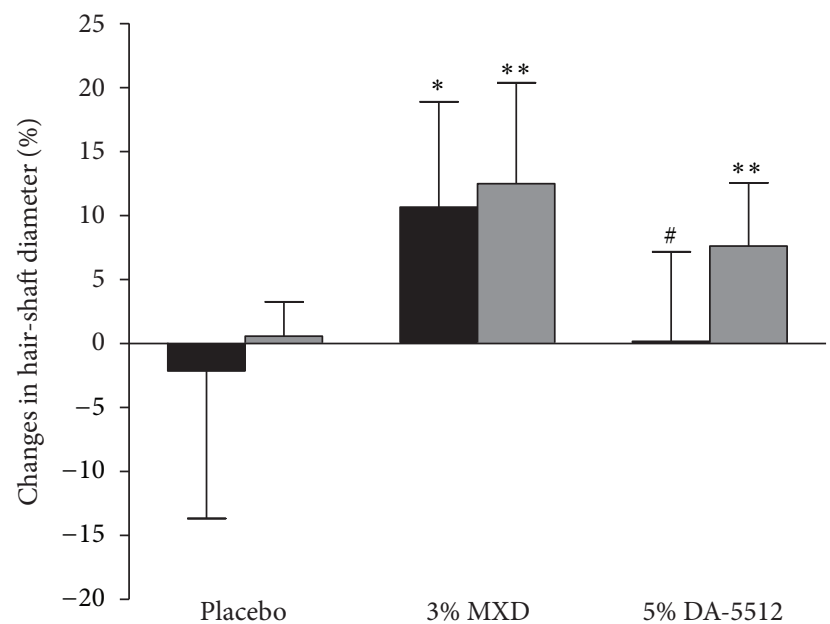

0-8 weeks

$8-16$ weeks

(a)

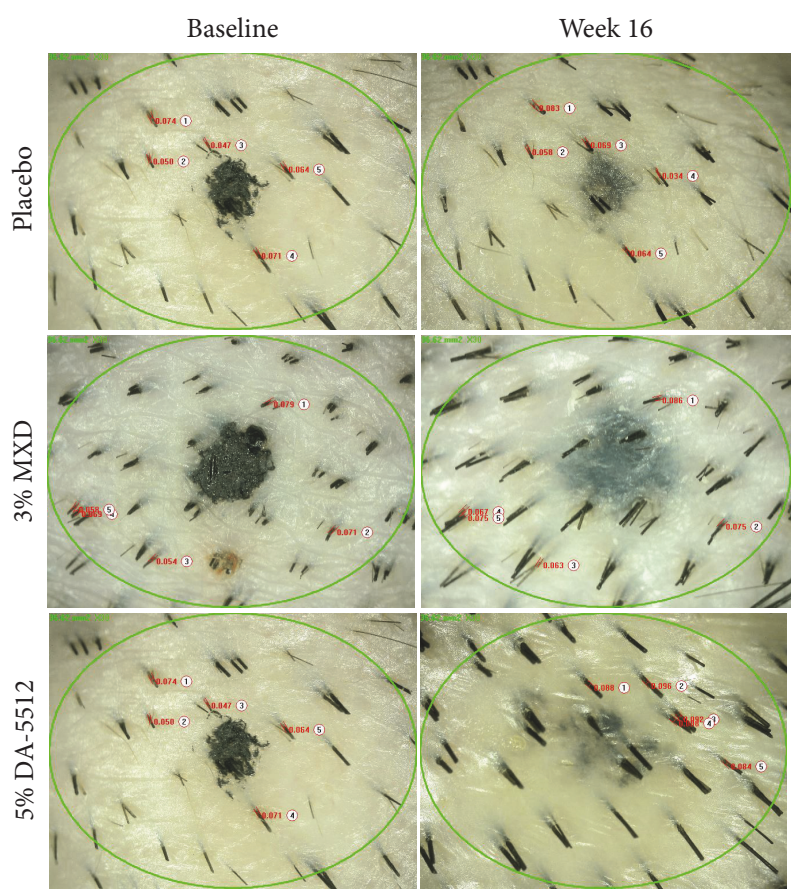

(b)

FIgURE 7: (a) Changes in hair-shaft diameter through days $0-8$ and $8-16$ after treatment with placebo, 3\% MXD, or 5\% DA-5512. All values are expressed as the mean $\pm \mathrm{SD} .{ }^{*} p<0.05$ and ${ }^{* *} p<0.01$ versus placebo, and ${ }^{\#} p<0.05$ versus 3\% MXD. (b) Folliscopy of hair-shaft diameters at baseline and after 16 weeks of treatment with placebo, $3 \% \mathrm{MXD}$, or 5\% DA-5512.

are shown in Figure 8. The rates after 8 and 16 weeks after treatment with $3 \% \operatorname{MXD}(0.039 \pm 0.049$ and $0.039 \pm 0.085$, resp.) and with 5\% DA-5512 (0.035 \pm 0.078 and $0.134 \pm 0.174$, resp.) were significantly higher $(p<0.05)$ compared with those of the placebo group $(-0.021 \pm 0.043$ and $-0.007 \pm 0.025)$. The performance of $5 \%$ DA-5512 was superior to that of $3 \%$ topical MXD after 16 weeks $(p<0.05)$.

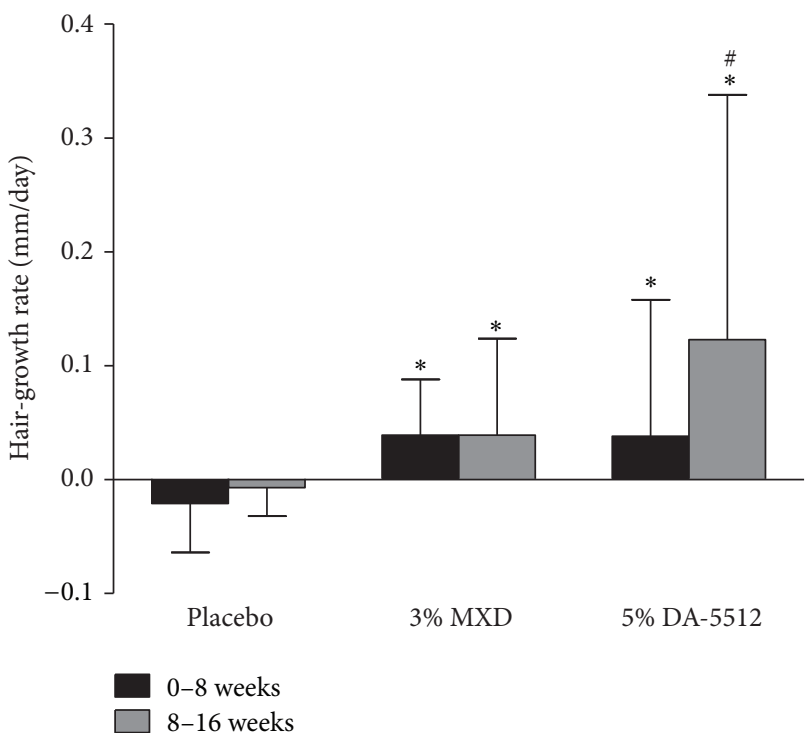

FIgURE 8: Changes in hair-growth rate through days 0-8 and 8-16 after treatment with placebo, $3 \% \mathrm{MXD}$, or $5 \% \mathrm{DA}-5512$. All values are expressed as the mean $\pm \mathrm{SD} .{ }^{*} p<0.05$ versus placebo. ${ }^{*} p<0.05$ versus 3\% MXD.

3.3.4. Investigator's Assessment Score of Hair Density. An investigator, uninformed of the nature of the study, assessed hair density using a standardized 7-point mean score. As shown in Figure 9, the mean scores for hair densities of the $3 \%$ MXD group (each $0.571 \pm 0.787,0.286 \pm 0.488$ ) and the $5 \%$ DA-5512 group (each $0.500 \pm 0.756,0.500 \pm 0.535$ ) were significantly higher compared with those of the placebo group (each $-0.250 \pm 0.463,-0.875 \pm 0.641)$ after $8(p<0.05)$ and 16 weeks $(p<0.01)$, respectively. Most subjects in the $3 \%$ MXD and 5\% DA-5512 groups exhibited slight or moderate improvement in hair-density assessment scores.

3.3.5. Clinical Response. Clinical responses were assessed via an investigator's assessment and each patient's selfassessment of the efficacy of the treatment according to hair growth, hair density, or satisfaction according to a 10-point scale, and each patient's self-assessment of scalp condition used a standard 5-point rating scale (Table 2). No significant differences were observed among the three groups at the end of week 16. The self-assessment scores of the subjects' scalp conditions were based on improvement of horny skin, itching, excess sebum, and dandruff, and the satisfaction scores showed no significant differences between the three groups.

3.3.6. Safety Evaluation. No product-related adverse effects were exhibited by subjects who received topical 5\% DA-5512, $3 \% \mathrm{MXD}$, or placebo (data not shown).

\section{Discussion}

The HF is a sac or a structure in the skin, which produces hair through a three-phase growth cycle as follows: anagen, catagen, and telogen [30]. HF morphogenesis involves the 
TABLE 2: Investigator's assessment and patients' self-assessment of clinical responses.

\begin{tabular}{|c|c|c|c|}
\hline \multirow{2}{*}{ End point } & \multicolumn{3}{|c|}{ Group } \\
\hline & Placebo & $3 \% \mathrm{MXD}$ & 5\% DA-5512 \\
\hline \multicolumn{4}{|c|}{ Efficacy assessment scores ${ }^{(1)}$} \\
\hline Hair growth & $6.875 \pm 1.553$ & $6.857 \pm 1.574$ & $6.250 \pm 1.282$ \\
\hline Hair diameter & $6.375 \pm 1.598$ & $5.143 \pm 1.574$ & $5.750 \pm 1.389$ \\
\hline \multicolumn{4}{|l|}{ Satisfaction $^{(2)}$} \\
\hline Hair line styling & $6.500 \pm 1.604$ & $5.429 \pm 0.787$ & $5.750 \pm 1.389$ \\
\hline Hair condition & $5.875 \pm 2.900$ & $5.857 \pm 1.574$ & $5.750 \pm 2.816$ \\
\hline Hair product & $6.375 \pm 2.264$ & $5.571 \pm 1.272$ & $5.875 \pm 1.356$ \\
\hline \multicolumn{4}{|l|}{ Scalp condition ${ }^{(3)}$} \\
\hline Keratin & $1.375 \pm 0.744$ & $1.857 \pm 0.690$ & $1.625 \pm 0.916$ \\
\hline Pruritus & $1.785 \pm 0.164$ & $2.000 \pm 0.816$ & $1.500 \pm 1.195$ \\
\hline Sebum & $1.378 \pm 0.954$ & $1.857 \pm 0.900$ & $1.875 \pm 0.641$ \\
\hline Dandruff & $1.125 \pm 0.991$ & $1.429 \pm 0.787$ & $1.250 \pm 1.035$ \\
\hline
\end{tabular}

downward migration of bulge stem cells and their entry into the matrix where they proliferate and differentiate to form HFs [31]. During anagen, follicles are long and straight and increase in size and number to produce an entire hair shaft. During catagen and telogen, the follicles reset and generate stem cells. When stem cells in the HFs are activated in response to the signal to start the next growth phase, they start a new anagen phase and produce a new hair shaft [32]. This process is clinically important, because many patients with hair disorders suffer from undesired alterations in HF cycling [33].

Cultured normal hDPCs, a hair-follicle organ culture model, and hair and skin specimens of mice were used to determine the effects of DA-5512. DA-5512 concentrations from 10 to 100 ppm enhanced the viability of hDPCs; however, $>10,000$ ppm was toxic. High concentrations overstimulate hair-follicle metabolism to cause extensive consumption of energy reserves, exhaustion of proliferative capacity, and inhibition of hair-shaft elongation. Further, the mechanism responsible for the stimulatory effects of DA-5512 on hairfollicle growth is unknown. To investigate the proliferative activity of hDPCs in response to DA-5512, we chose a dose of $100 \mathrm{ppm}$. We found that Ki-67 levels increased in hDPCs upon treatment with DA-5512 or MXD. Ki-67, which detects nuclei in matrix keratinocytes, is an indicator of the proliferation of anagen hair follicles [34, 35]. Therefore, DA5512 may promote hair regrowth, because it increased the proliferation of hDPCs.

C57BL/6 mice are used to screen for the effects of hair-growth-promoting agents [36]. C57BL/6 mice lack melanocytes in their skin, and therefore melanogenesis occurs only in hair follicles, and melanins are produced only during anagen phase [37]. Therefore, melanogenesis is strongly related to the hair-growth cycle in these mice. The skin of C57B1/6 mice is pale pink in the resting telogen phase, becomes dark gray or black during active hair growth in the anagen phase, and returns to pale pink in the catagen phase when hair growth ceases and the skin transitions back to the telogen phase. Hair growth of the DA-5512-treated group was higher compared with that of the NC group and was similar to that of the 3\% MXD-treated group. DA-5512 significantly shortened the time for skin darkening and hair growth and increased hair length and weight, indicating that DA-5512 stimulated the anagen phase of the hair-growth cycle. Histological analysis revealed that the number of hair follicles in the DA-5512-treated group was significantly higher compared with that of the NC group, suggesting that DA5512 may elicit its hair-growth-promoting activity through an increase in hair-follicle number and through stimulation of anagen.

We conducted a double-blind, placebo-controlled, randomized clinical trial to evaluate the ability of DA-5512 to promote hair growth in patients with pattern hair loss. Statistically significant increases in hair density, hair diameter, hair-growth rate, and investigator's assessment scores of hair density characterized the 3\% MXD and 5\% DA-5512 groups compared with those of the placebo group after 16 weeks. Our data reveal that topical treatment with 5\% DA-5512 led to increases in hair density and diameter, which was significantly greater at 16 weeks compared with 8 weeks, suggesting that using DA-5512 continuously for longer times may have beneficial effects on hair loss.

Hair diameter and density tend to decrease with hair loss, suggesting that because DA-5512 treatment increased hair diameter and density, it may enhance hair growth or slow the progression of hair loss. Interestingly, the patient's selfassessment scores did not demonstrate a significant outcome for patterned hair loss. However, an overall improvement in hair growth or density, in scalp condition (horny skin, itching, excess sebum, and dandruff), and in satisfaction with 5\% topical DA-5512 compared with placebo indicated a definite advantage of DA-5512 for treating patterned hair loss.

Several varieties of plants are used to prepare DA-5512 to prevent or treat hair loss, to nourish hair, and to improve the esthetic properties of hair. These plants produce inhibitors of $5 \alpha$-reductase activity. Green tea is rich in the flavanol group 


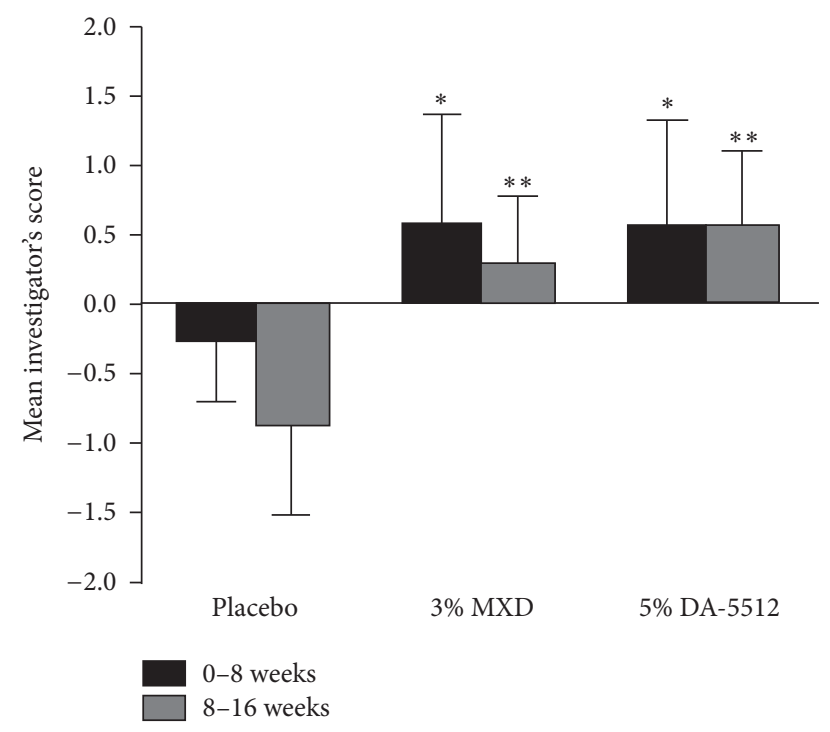

(a)

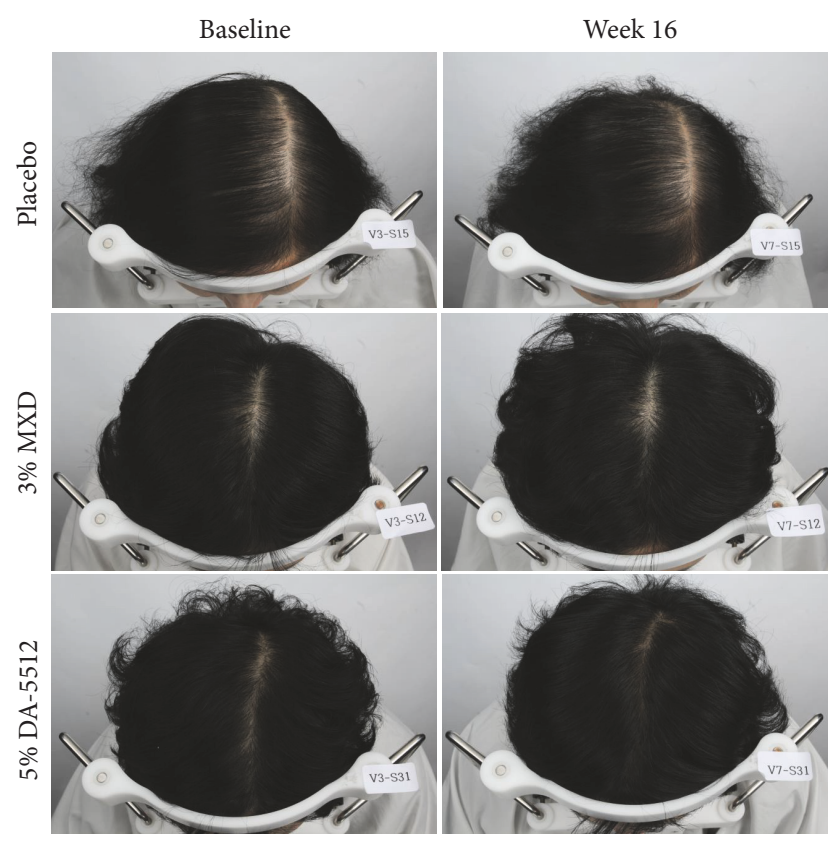

(b)

FIGURE 9: (a) Investigator's assessment score through days $0-8$ and 8-16 after treatment with placebo, 3\% MXD, or 5\% DA-5512. All values are expressed as the mean $\pm \mathrm{SD} .{ }^{*} p<0.05$ and ${ }^{* *} p<$ 0.01 versus placebo. (b) Representative photographs of patients at baseline and week 16 .

of polyphenols called catechins, and green tea polyphenols positively affect hair growth and follicle health. Possible mechanisms of action include inhibition of apoptosis, radioprotection of follicle cells, antioxidant activity, and potential follicular inhibition of TGF- $\beta 1$ [38].

Green tea is a popular herbal remedy that contains catechins that may inhibit $5 \alpha$-reductase. EGCG, the most abundant catechin found in green tea, prevents or treats androgenetic alopecia by selectively inhibiting $5 \alpha$-reductase activity [39]. P. emblica L. and Zingiber officinale promote hair growth by inhibiting $5 \alpha$-reductase activity [19] in vitro and in vivo. Moreover, Puerariae flos inhibits testosterone $5 \alpha$-reductase in vitro and exhibits antiandrogenic activity that was detected using a hair-growth assay in testosteronesensitive male C57Black/6NCrSlc mice [40]. $5 \alpha$-Reductase catalyzes the conversion of testosterone into dihydrotestosterone (DHT) that combines with the same androgenic receptor to form a conjugate, which leads to hair-follicle miniaturization and then to telogen [41]. Thus, DA-5512 might influence hair growth by inhibiting $5 \alpha$-reductase activity.

A preclinical study using a Pueraria thunbergiana (pine) needle extract revealed its role in the improvement of hair growth in C57BL/6 mice [42]. This study shows that pine needle extract treatment increases the levels of IGF-1 and VEGF, which play a role in increasing the size of hair follicles, in angiogenesis in the outer root sheet, and inhibit TGF- $\beta 1$, which disrupts hair growth. High levels of TGF- $\beta 1$ stimulate androgen or DHT production, which inhibits the growth of keratinocytes in dermal papilla. Pine needle extract is an antioxidant that is a potent scavenger of free radicals. Further, it inhibits the production of reactive oxygen species by stimulating superoxide dismutase and glutathione peroxidase to promote hair growth and to treat hair loss.

The antihypertensive effects of the T. terrestris fruit are similar to those of MXD, and its beneficial effects are attributed to vasodilator activity [25]. The mechanism of the effect of T. terrestris fruit on human hair growth is unknown, but it may act as a growth factor for hair roots. Therefore, these properties suggest that the various components of DA5512 might account for its hair-growth-promoting activity that enhances hair health.

In summary, we show here that in a mouse model DA-5512 promoted the proliferation of human DPCs and stimulated hair growth. Further, a clinical study detected enhanced hair growth or slowed progression of hair loss upon treatment of patients with DA-5512. However, the mechanisms of DA-5512 associated with its effect of hair loss remain to be determined and therefore require further study.

In conclusion, the natural components of DA-5512 might influence hair-growth-promoting activity and enhance hair health and can therefore be considered an effective option for treating hair loss.

\section{Conflicts of Interest}

There is a conflict of interest regarding the publication of this manuscript. Jae Young Yu, Hyoung Geun Park, Miwon Son, and Joon-Ho Jun are employees of the Dong-A ST Research Institute. The other authors have no conflicts of interest to declare.

\section{Authors' Contributions}

Jae Young Yu and Biki Gupta contributed equally to this work.

\section{Acknowledgments}

This research was supported by the Yeungnam University research grants in 2016. 


\section{References}

[1] M. Alfonso, H. Richter-Appelt, A. Tosti, M. S. Viera, and M. García, "The psychosocial impact of hair loss among men: A Multinational European Study," Current Medical Research and Opinion, vol. 21, no. 11, article 2861, pp. 1829-1836, 2005.

[2] M. B. Chartier, D. M. Hoss, and J. M. Grant-Kels, "Approach to the adult female patient with diffuse nonscarring alopecia," Journal of the American Academy of Dermatology, vol. 47, no. 6, pp. 809-818, 2002.

[3] S. Shrivastava, "Diffuse hair loss in an adult female: approach to diagnosis and management," Indian Journal of Dermatology, Venereology and Leprology, vol. 75, no. 1, pp. 20-28, 2009.

[4] J. V. Schmitt, C. F. Ribeiro, F. H. D. M. de Souza, E. B. D. Siqueira, and F. R. L. Bebber, "Hair loss perception and symptoms of depression in female outpatients attending a general dermatology clinic," Anais Brasileiros de Dermatologia, vol. 87, no. 3, pp. 412-417, 2012.

[5] A. E. Buhl, D. J. Waldon, T. Kawabe, and J. M. Holland, "Minoxidil stimulates mouse vibrissae follicles in organ culture," Journal of Investigative Dermatology, vol. 92, no. 3, pp. 315-320, 1989.

[6] A. E. Buhl, "Minoxidil's action in hair follicles," Journal of Investigative Dermatology, vol. 96, pp. S73-S74, 1991.

[7] J. Kubilus, J. C. Kvedar, and H. P. Baden, "Effect of minoxidil on pre- and postconfluent keratinocytes," Journal of the American Academy of Dermatology, vol. 16, no. 3, pp. 648-652, 1987.

[8] M. P. Philpott, D. A. Sanders, and T. Kealey, "Whole hair follicle culture," Dermatologic Clinics, vol. 14, no. 4, pp. 595-607, 1996.

[9] H. Ebner and E. Müller, "Allergic contact dermatitis from minoxidil," Contact Dermatitis, vol. 32, no. 5, pp. 316-317, 1995.

[10] A. Tosti, F. Bawjazzi, M. P. de Padova, and et al., "Contact dermatitis to Minoxidil," Contact Dermatitis, vol. 13, pp. 275-276, 1985.

[11] C. Wilson, V. Walkden, S. Powell, S. Shaw, J. Wilkinson, and R. Dawber, "Contact dermatitis in reaction to $2 \%$ topical minoxidil solution," Journal of the American Academy of Dermatology, vol. 24, no. 4, pp. 661-662, 1991.

[12] M. Iorizzo, C. Vincenzi, S. Voudouris, B. M. Piraccini, and A. Tosti, "Finasteride treatment of female pattern hair loss," Archives of Dermatology, vol. 142, no. 3, pp. 298-302, 2006.

[13] D. Rathnayake and R. Sinclair, "Innovative use of spironolactone as an antiandrogen in the treatment of female pattern hair loss," Dermatologic Clinics, vol. 28, no. 3, pp. 611-618, 2010.

[14] E. A. Olsen, "Androgenetic alopecia," in Disorders of Hair Growth: Diagnosis and Treatment, E. A. Olsen, Ed., pp. 257-287, McGraw Hill, New York, NY, USA, 1993.

[15] S. Hsu, W. B. Bollag, J. Lewis et al., "Green tea polyphenols induce differentiation and proliferation in epidermal keratinocytes," Journal of Pharmacology and Experimental Therapeutics, vol. 306, no. 1, pp. 29-34, 2003.

[16] O. S. Kwon, J. H. Han, H. G. Yoo et al., "Human hair growth enhancement in vitro by green tea epigallocatechin-3-gallate (EGCG)," Phytomedicine, vol. 14, no. 7-8, pp. 551-555, 2007.

[17] M. S. Baliga and J. J. Dsouza, "Amla (Emblica officinalis Gaertn), a wonder berry in the treatment and prevention of cancer," European Journal of Cancer Prevention, vol. 20, no. 3, pp. 225239, 2011.

[18] R. S. Thakur, H. S. Puri, and A. Husain, Major Medicinal Plants of India, Central Institute of Medicinal and Aromatic Plants, Lucknow, India, 1989.
[19] N. Kumar, W. Rungseevijitprapa, N.-A. Narkkhong, M. Suttajit, and C. Chaiyasut, " $5 \alpha$-reductase inhibition and hair growth promotion of some Thai plants traditionally used for hair treatment," Journal of Ethnopharmacology, vol. 139, no. 3, pp. 765-771, 2012.

[20] H. Wang, X. D. Gao, G. C. Zhou, L. Cai, and W. B. Yao, "In vitro and in vivo antioxidant activity of aqueous extract from Choerospondias axillaris fruit," Food Chemistry, vol. 106, no. 3, pp. 888-895, 2008.

[21] K. H. Wong, G. Q. Li, K. M. Li, V. Razmovski-Naumovski, and K. Chan, "Kudzu root: traditional uses and potential medicinal benefits in diabetes and cardiovascular diseases," Journal of Ethnopharmacology, vol. 134, no. 3, pp. 584-607, 2011.

[22] T. Yamazaki, T. Hosono, Y. Matsushita et al., "Pharmacological studies on puerariae flos. IV: effects of pueraria thomsonii dried flower extracts on blood ethanol and acetaldehyde levels in humans," International Journal of Clinical Pharmacology Research, vol. 22, pp. 23-28, 2002.

[23] S. Bhargava, K. Dhabhai, A. Batra, and et al., "Zingiber officinale: chemical and phytochemical screening and evaluation of its antimicrobial activities," Journal of Chemical and Pharmaceutical Research, vol. 4, pp. 360-364, 2012.

[24] J. Heinerman, Heinerman's Encyclopedia of Fruits, Vegetables and Herbs, Parker Publishing Co., New York, NY, USA, 1988.

[25] S. Hashim, T. Bakht, K. B. Marwat et al., "Medical properties, phytochemistry and pharmacology of tribulus terrestris L. (zygophyllaceae)," Pakistan Journal of Botany, vol. 46, pp. 399404, 2014.

[26] A. G. Messenger, "The culture of dermal papilla cells from human hair follicles," British Journal of Dermatology, vol. 110, no. 6, pp. 685-689, 1984.

[27] M. P. Philpott, D. Sanders, G. E. Westgate, and T. Kealey, "Human hair growth in vitro: a model for the study of hair follicle biology," Journal of Dermatological Science, vol. 7, no. 1, pp. S55-S72, 1994.

[28] T. Mosmann, "Rapid colorimetric assay for cellular growth and survival: application to proliferation and cytotoxicity assays," Journal of Immunological Methods, vol. 65, no. 1-2, pp. 55-63, 1983.

[29] J. H. Han, O. S. Kwon, J. H. Chung, K. H. Cho, H. C. Eun, and K. H. Kim, "Effect of minoxidil on proliferation and apoptosis in dermal papilla cells of human hair follicle," Journal of Dermatological Science, vol. 34, no. 2, pp. 91-98, 2004.

[30] L. Li and T. Xie, "Stem cell niche: structure and function," Annual Review of Cell and Developmental Biology, vol. 21, pp. 605-631, 2005.

[31] H. Ogawa and M. Hattori, "Regulation mechanisms of hair growth," in Current Problems in Dermatology, vol. 11, pp. 159170, Karger Publishers, Basel, Switzerland, 1983.

[32] R. Paus and K. Foitzik, "In search of the 'hair cycle clock': a guided tour," Differentiation, vol. 72, no. 9-10, pp. 489-511, 2004.

[33] K. Datta, A. T. Singh, A. Mukherjee, B. Bhat, B. Ramesh, and A. C. Burman, "Eclipta alba extract with potential for hair growth promoting activity," Journal of Ethnopharmacology, vol. 124, no. 3, pp. 450-456, 2009.

[34] C. G. Gho, J. E. F. Braun, C. M. L. J. Tilli, H. A. M. Neumann, and F. C. S. Ramaekers, "Human follicular stem cells: their presence in plucked hair and follicular cell culture," British Journal of Dermatology, vol. 150, no. 5, pp. 860-868, 2004.

[35] X. Xu, S. Lyle, Y. Liu et al., "Differential expression of cyclin D1 in the human hair follicle," American Journal of Pathology, vol. 163, pp. 969-978, 2003. 
[36] A. Slominski, R. Paus, P. Plonka et al., "Melanogenesis during the anagen-catagen-telogen transformation of the murine hair cycle," Journal of Investigative Dermatology, vol. 102, no. 6, pp. 862-869, 1994.

[37] L. Alonso and E. Fuchs, “The hair cycle," Journal of Cell Science, vol. 119, no. 3, pp. 391-393, 2006.

[38] S. M. Patil, G. N. Sapkale, U. S. Surwase et al., "Herbal medicines as an effective therapy in hair loss-a review," Research Journal of Pharmaceutical, Biological and Chemical Sciences, vol. 1, pp. 773-781, 2010.

[39] R. A. Hiipakka, H.-Z. Zhang, W. Dai, Q. Dai, and S. Liao, "Structure-activity relationships for inhibition of human $5 \alpha-$ reductases by polyphenols," Biochemical Pharmacology, vol. 63, no. 6, pp. 1165-1176, 2002.

[40] B. Zhang, R.-W. Zhang, X.-Q. Yin et al., "Inhibitory activities of some traditional Chinese herbs against testosterone $5 \alpha$ reductase and effects of Cacumen platycladi on hair re-growth in testosterone-treated mice," Journal of Ethnopharmacology, vol. 177, pp. 1-9, 2016.

[41] D. Rathnayake and R. Sinclair, "Male androgenetic alopecia," Expert Opinion on Pharmacotherapy, vol. 11, no. 8, pp. 12951304, 2010.

[42] B. Shin, "Research into the effects of pine-needle extracts on the hair growth factors and physiological changes," Journal of the Korean Society of Cosmetology, vol. 19, pp. 588-595, 2013. 


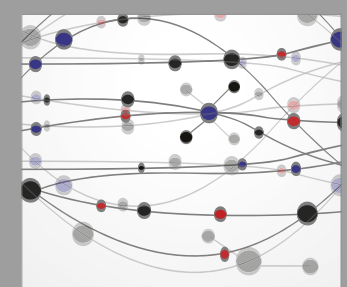

The Scientific World Journal
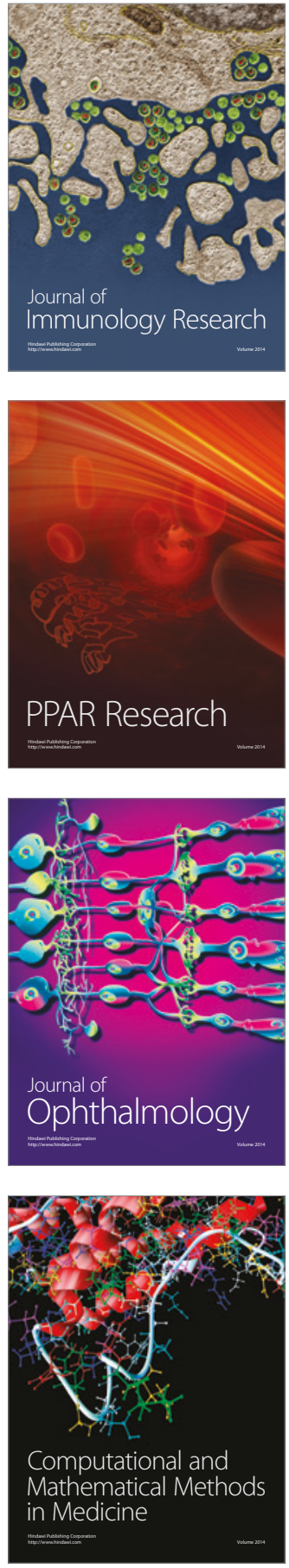

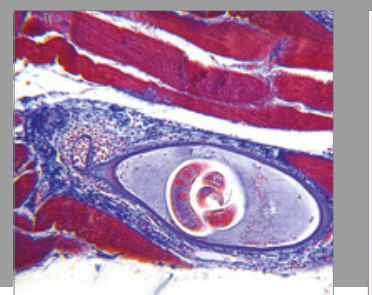

Gastroenterology Research and Practice
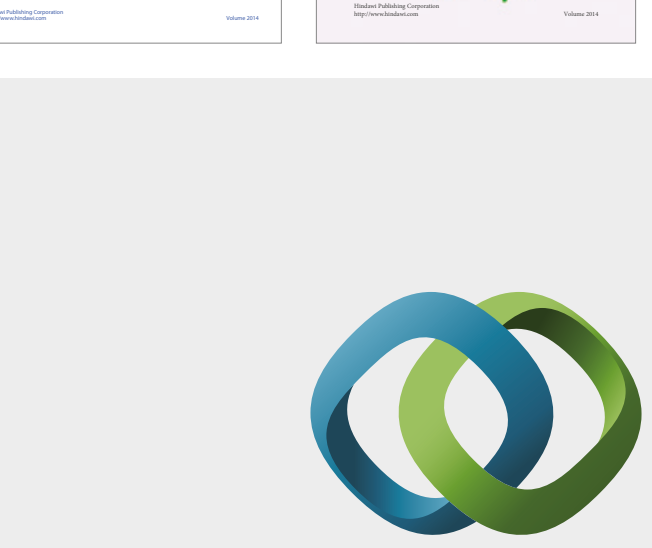

\section{Hindawi}

Submit your manuscripts at

https://www.hindawi.com
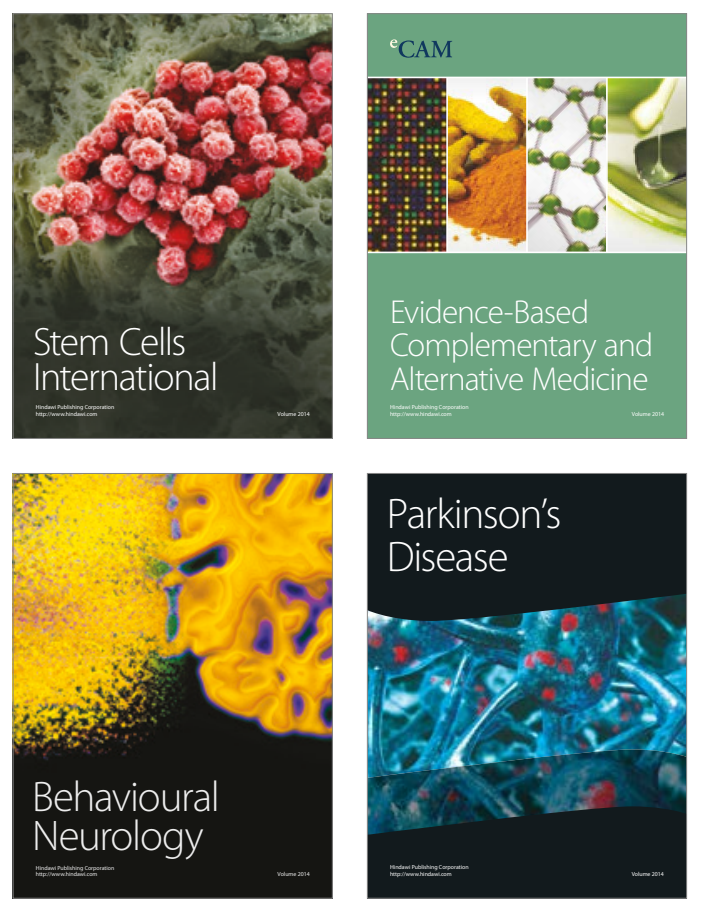
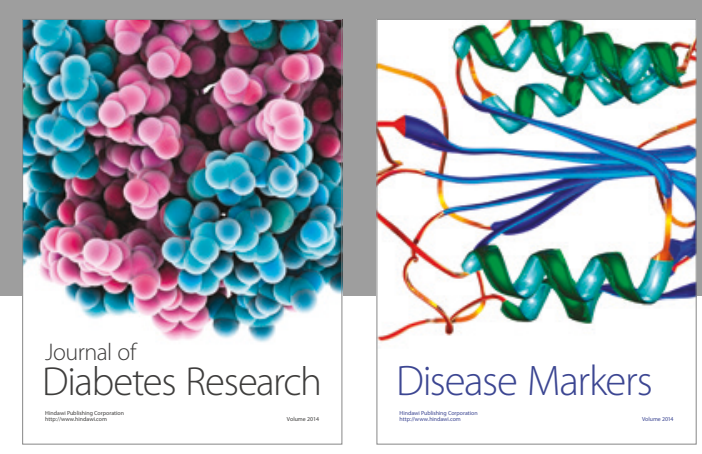

Disease Markers
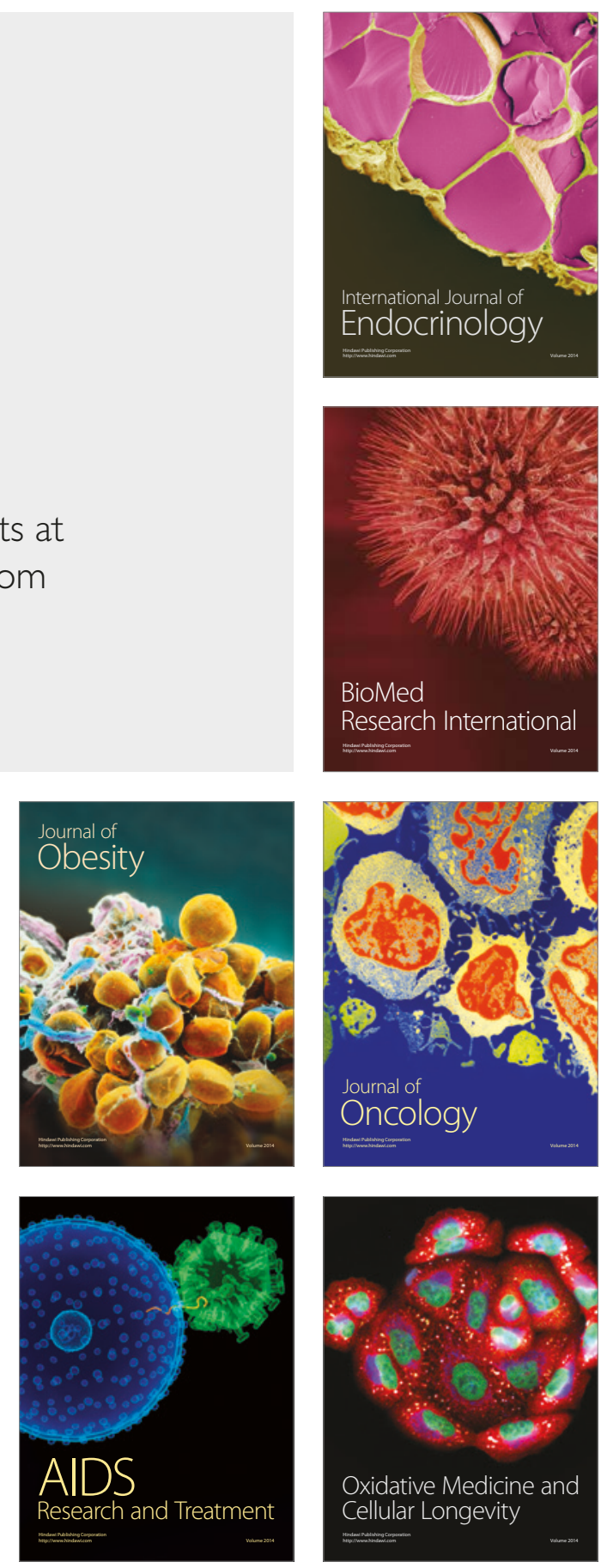